\title{
DE LA FILOSOFÍA DE LA LOCURA A LA HIGIENE DEL ALMA. JOSEPH DAQUIN (1732-1815)
}

\author{
Rafael Huertas \\ Departamento de Historia de la Ciencia \\ Instituto de Historia, CSIC - España \\ rafael.huertas@cchs.csic.es
}

Recibido: 12 de abril de 2015; Aceptado: 1 de septiembre de 2015.

Cómo citar este artículo/Citation: Huertas, Rafael (2015), "De la filosofía de la locura a la higiene del alma. Joseph Daquin (17321815)", Asclepio, 67 (2): p106. doi: http://dx.doi.org/10.3989/asclepio.2015.24

RESUMEN: La Philosophie de la folie (1791; 2a ed.: 1804) constituye uno de los antecedentes fundamentales de lo que más tarde se denominaría tratamiento moral. Su autor, Joseph Daquin, fue médico en el Hospice des Incurables de la ciudad de Chambèry (antiguo ducado de Saboya; Reino de Piamonte-Cerdeña) desde 1788. La obra recoge sus reflexiones sobre la naturaleza y las causas de la locura y se sitúa con claridad en las corrientes filantrópicas del fin de las luces; defiende en todo momento el trato humanitario a los orates, revisa los tratamientos tradicionales e introduce nuevas formas de terapia, entre las que destaca la prudencia hipocrática (e ilustrada) de la vis medicatrix naturae y la dieta, entendida como régimen de vida, como higiene del alma. En definitiva, un análisis pormenorizado de la obra de Daquin, omitida por sus contemporáneos parisinos, nos permitirá valorar y reivindicar su importancia en el origen del alienismo.

PALABRAS CLAVE: Joseph Daquin; Philippe Pinel; Ilustración; Tratamiento Moral; Alienismo; Locura.

\section{FROM THE PHILOSOPHY OF MADNESS TO THE HEALTH OF THE SOUL. JOSEPH DAQUIN (1731-1815)}

ABSTRACT: La Philosophie de la folie (1791; 2nd ed.: 1804) constitutes an essential precursor of what would later come to be known as moral treatment. Its author, Joseph Daquin, was a physician at the Hospice des Incurables patients in the city of Chambèry (formerly the Duchy of Savoy; Kingdom of Piedmont-Sardinia) from 1788. The work contains his thoughts on nature and the causes of madness, and is clearly in tune with the philanthropic trends of the end of the Enlightenment. It defends humane treatment of lunatics at all times, revises traditional treatments and introduces new forms of therapy. Daquin emphasizes the Hippocratic (and enlightened) prudence of the vis medicatrix naturae and diet, understood as a regimen for life, as the health of the soul. In short, a detailed analysis of the work by Daquin, overlooked by his Parisian contemporaries, will enable us to evaluate and affirm his importance to the origins of alienism.

KEY WORDS: Joseph Daquin; Philippe Pinel; Enlightenment; Moral Treatment; Alienism; Madness. 
Una década antes del famoso Traité médico-philosophique sur l'alienation mentale, ou la manie (1801) de Philippe Pinel, se publicó La Philosophie de la folie $(1791)^{1}$ del médico saboyano Joseph Daquin. Se trata de una obra importante y sin duda iniciadora de lo que muy poco tiempo después sería conocido como tratamiento moral. La obra de Daquin ha sido escasamente considerada por la historiografía, más allá de algunos trabajos monográficos ya antiguos (Nyffeler' 1961; Caron, 1964) o de las breves, aunque obligadas, referencias a su figura en obras más o menos generales de historia de la psiquiatría (Gauchet y Swain, 1980, pp. 413-422; Shorter, 1997, pp. 25-26). Joseph Daquin es, habitualmente, presentado -en ocasiones con tintes nacionalistas (Padovani, 1927; Ferrio, $1954)^{2}$ - como pionero y precursor del tratamiento moral y, por extensión, del movimiento alienista, aunque pronto eclipsado por el esplendor de la obra pineliana. A ello debió contribuir el propio Pinel, al ignorar absolutamente su aportación en contraste con el reconocimiento que siempre mostró hacia la obra de Alexander Crichton. No deja de resultar significativo que la segunda edición de La Philosophie de la folie, publicada en 1804, esté dedicada "Au Docteur Pinel» y que, junto al elogio al Traité, Daquin se lamente de la ausencia en el mismo de cualquier alusión a la primera edición de su libro.

No cabe duda de que entre los «primeros alienistas», Daquin es posiblemente el más olvidado, menos influyente que Crichton (Weiner, 1999; Charland, 2008), como ya he indicado, y menos conocido que el florentino Vincenzo Chiarugi (Vanni et al, 1999). Sin embargo, merece la pena revisitar su obra, en un intento no tanto de reivindicar su importancia en la historia de la psiquiatría, y mucho menos para oponer su figura a la del gran Pinel, pero si para valorar algunos aspectos de su obra que pueden ofrecer elementos interesantes en la reflexión sobre el nacimiento del alienismo.

\section{UN HIJO DE LA ILUSTRACIÓN}

Tras estudiar medicina en Turín, Daquin frecuentó las facultades de Montpellier y París, desde donde regresó a Chambéry, su ciudad natal, a ejercer primero en l'Hôtel-Dieu y, más tarde, a partir de 1788, en l'Hospice des Incurables, donde tuvo a su cargo a unos veinte enfermos mentales. Su preocupación por dichos pacientes y la experiencia adquirida con ellos, le llevo a escribir y publicar en 1791 la obra citada anteriormente. Daquin se sitúa con claridad en las corrientes filantrópicas del fin de las luces, defendiendo el trato humanitario a los orates, revisando los tratamientos tradicionales e introduciendo propuestas terapéuticas ciertamente novedosas.

El primer párrafo de La Philosophie de la folie contiene, en sí mismo, la voluntad programática de aunar medicina y filantropía:

Bajar a las celdas para observar y describir la economía animal desorganizada es una empresa desoladora. ¡Cuán triste es la ciencia que aborda la locura! Quien la ejerce se ve obligado a examinar a individuos de su misma naturaleza que - no siendo sin embargo como él- parecen situarse en un estado intermedio entre el hombre y el bruto. La medicina precisamente es la ciencia que, por desgracia, nunca colma su deseo de aliviar los males que sin cesar nos acechan. Y siendo la profesión de médico muy penosa, mucho más lo será si debe visitar a personas cuyas funciones intelectuales están alteradas, si tiene que perder la razón, por así decirlo, con ellas y escuchar lo más extraordinario que el espíritu humano pueda engendrar $y$, sobre todo, si trata de cuidarlos, de encontrar remedios, quizá no para sanarlos completamente, pero al menos para aliviarlos y dulcificar una suerte acaso mucho menos compasible de lo que podríamos creer. Porque no poseyendo el verdadero, el justo sentimiento de lo que son, los locos se muestran incapaces de reflexionar sobre su estado y de apreciar el alcance del infortunio en el que están sumidos (Daquin, 1791, p. 1).

Se lamenta de la ausencia de tradición en la observación, estudio y tratamiento de los locos, lo que achaca al prejuicio de muchos médicos que, concibiendo la enfermedad mental como incurable, pensaban que «cuando un hombre da signos de demencia hay que encerrarlo enseguida porque puede perjudicar a sus semejantes o porque ya no puede servir para nada» (Daquin, 1791, p. 5).

J. Daquin entiende la locura como «lo contrario de la razón» (Daquin, 1791, p. 7-8) -lo que para un ilustrado no deja de tener un significado muy especial- y amplía su definición, explicando que «La locura en general es ese estado en el que el ejercicio de las operaciones del alma o del espíritu no se realiza completamente ni sigue siempre las leyes del orden natural» (Daquin, 1791, p. 7). Se trata, como puede apreciarse, de una definición filosófica que está próxima a la idea cartesiana del dominio de la razón sobre la materia, siendo la razón, aquella «facultad que la naturaleza ha provisto a cada hombre para conocer la verdad» (Daquin, 1791, p. 8). Junto a la 
razón, admite la existencia de otras dos operaciones o facultades del alma: la memoria y la imaginación, pero éstas se ordenan para que la primera -la razón- pueda reconocer la verdad ${ }^{3}$.

Las causas de esta "falta de razón» pueden, según nuestro médico, ser físicas y morales; entre las primeras destacan las «alteraciones orgánicas del cerebro», aunque reconoce que

"como las funciones de esta víscera están todavía poco definidas y como faltan conocimientos sobre este tema en medicina, todavía no se ha podido descubrir la influencia que las distintas partes de este órgano ejerce sobre cada una de sus operaciones» (Daquin, 1791, p. 16).

Pero, junto a las inflamaciones, compresiones, rigidez o laxitud del cerebro y sus envolturas, hay que tener en cuenta la fuerza que adquiere el temperamento con el paso de los años, las pasiones de las que el ser humano es prisionero, pasiones que, al "estrechar los nudos que unen las ideas, al aflojarlos o interrumpirlos del todo, nos hacen caer en la locura» (Daquin, 1791, p. 18). Digno alumno de Montpellier, la pasión es para Daquin, como lo era para el primer Esquirol (1805), la causa de la enfermedad mental (Huertas, 2008). Habrá, pues, que curar el alma como filósofo, tendiendo un puente entre las ciencias médicas y las ciencias morales, para que el médico, convertido en filósofo, pueda curar el cuerpo pero también el alma.

Se trata, claro está, de una percepción de las pasiones agitadas o desordenadas en tiempos de desorden. La religión o la codicia, el orgullo o la ambición pueden perturbar la razón. Las violentas luchas de la burguesía por sustituir a la nobleza, consiguiendo dinero, poder y honor se unen a las viejas angustias místicas. Sin embargo, como nos advierte José Luis Peset, "la ciencia ha sustituido las creencias heredadas, colocando a la diosa razón en un nuevo altar. Siguiendo a Tissot y éste a Rousseau, el saber también ha subvertido el orden natural y con él las mentes» (Peset, 2000, p. 9).

Las pasiones pasan así a ser entendidas como fenómenos de la economía animal, susceptibles de ser observadas y estudiadas científicamente y al margen de cualquier especulación:

Cualquier tipo de pasión que afecta a los hombres puede considerarse la causa de su locura (...) el amor, los celos inseparables de este, la ira, la ambición, la venganza -que son pasiones ardientes- producen locos furiosos; mientras que la ternura paternal o filial, la de los esposos, la amistad (ese sentimiento dulce y apacible), los antojos, la religión, el estudio, la contemplación y las demás inclinaciones dulces, provocan por el contrario locos tranquilos, imbéciles, o causan locuras en las que el enfermo experimenta intervalos de tranquilidad, de buen sentido y razón (Daquin, 1791, p. 28).

Al hilo de estas afirmaciones, cabe indicar que Daquin propone una clasificación clínica de la locura un tanto rudimentaria en la que admite diferentes grados entre las clases de alienación; así, describe locos furiosos y tranquilos, extravagantes e imbéciles, insensatos y dementes. No se interesa demasiado por diferenciar manía y melancolía y se puede decir que tiene, al igual que sus contemporáneos Pinel o Chiarugi, una concepción «unitaria» de la alienación mental (Berrios y Beer, 1994; Huertas, 1999).

Sin embargo, en este proceso de medicalización de las pasiones no todos los comportamientos alejados de la razón fueron considerados patológicos. Daquin dedica algunas páginas a la «delicada cuestión» del suicidio que contrastan fuertemente con lo que serán las tendencias conceptuales sobre el mismo en el alienismo más inmediato. Para el saboyano el suicida no es un loco; es más, según su experiencia clínica, "los locos raramente atentan contra su vida» (Daquin, 1791, p. 92). Además, en la mayoría de las ocasiones, el acto suicida tiene unos ritos de preparación y ejecución que demuestran, en el sentir de Daquin, «ideas meditadas, estudiadas y tan bien unidas que anuncian un juicio muy sano y un razonamiento tan ajustado que en raras ocasiones, por no decir nunca, se encuentra en los locos» (Daquin, 1791, p. 95). El argumento es insistente y categórico: «el suicida no es un loco; es un cobarde y un vicioso, si consideramos la cobardía como un vicio del alma» (Daquin, 1791, p. 96). Cobardía, degradación, indigna debilidad, del que no es capaz de soportar las dificultades y sucumbe ante ellas.

Para el pensamiento ilustrado, el suicidio deja de ser paulatinamente un delito contra Dios y contra el ser humano para ser considerado como un «asunto de libertad individual». Es sabido que autores como Voltaire, Rousseau o Hume defendieron la libertad individual de las personas para disponer de su vida pero, a pesar de esta racionalización del acto suicida, resulta muy evidente su desasosiego hacia el mismo, entre otras cosas porque el suicida supone una afrenta a las ideas ilustradas y al tono -quizás falso o al menos matizado- optimista y progresista que se adscribe 
a la llustración. Dicho de otro modo, el suicida viene a representar el fracaso del proyecto ilustrado, siendo el espíritu romántico el que, posteriormente, entenderá el suicidio como un escape del dolor, la amargura y la desesperación (Cuevas, 2006, p. 13).

El alienismo francés sí establecerá una relación directa entre suicidio y enfermedad mental. Pinel describió una variedad de melancolía con una «inclinación irresistible al suicidio» (Pinel, 1801, p, XXVIII; p, 146 y ss), $y$, aunque no faltaron los autores que postularon que el suicidio debía ser considerado «en todos los casos» como resultado de la alienación mental (Chevrey, 1816), fue finalmente Esquirol el que, según la expresión de Berrios y Mohanna (1995, p. 614), estableció la «visión estándar» sobre la definición y clasificación del suicidio, admitiéndose la existencia de unos actos suicidas propiciados por la patología mental y de otros sin relación alguna con la locura. En todo caso, llama la atención la forma en que Daquin intuye y deja apuntado lo que muy pocos años más tarde constituiría un importante debate, mantenido durante todo el siglo XIX, entre los que consideraban, y los que no, que el suicido era «siempre» el acto de un alienado (Berrios y Mohanna, 1990).

Eran tiempos de fuertes conmociones sociales, de crisis políticas y de cambios culturales que pronto se relacionaron no solo con el aumento de los suicidios (Martínez-Pérez' 2001), sino con una nueva conceptuación de la locura en la que los viejos paradigmas se resistían a desaparecer y terminaron conviviendo con nuevos conocimientos que pugnaban por afianzarse. En la obra de Daquin encontramos, en este sentido, desde reminiscencias de ancestrales interpretaciones astrológicas de la locura; eso sí, suficientemente racionalizadas y naturalizadas, hasta el esbozo de un claro pensamiento anatomo-clínico.

\section{EN TORNO A LA CAUSALIDAD: DEL INFLUJO DE LA LUNA A LA LESIÓN ANATÓMICA}

El interés de Daquin por la influencia de los ciclos lunares sobre la locura proviene de la obra del abate Giuseppe Toaldo (1770), matemático, meteorólogo y astrónomo de Padua. Nuestro médico había traducido al francés el Ensayo meteorológico del científico italiano (Toaldo, 1784), en el que se apuntaba la relación entre las fases de la luna y «un gran número de enfermedades corporales» (Daquin, 1791, p. 83). Una correspondencia que Daquin pretende establecer también con las enfermedades mentales, recurriendo al seguimiento detallado de diez pacientes a los que «visitaba asiduamente con cada luna nueva, en cada pri- mer cuarto, en cada plenilunio y en cada último cuarto» (Daquin, 1791, p. 85). Una minuciosa observación empírica mediante la cual Daquin considera probado que la Luna ejerce una influencia real sobre la locura.

Siguiendo a Toaldo, define puntos "afirmativos» (luna nueva y cuarto creciente), de mayor influencia sobre la locura, y puntos «negativos» (luna llena y cuarto menguante), en los que dicha influencia es menor. Al contrario de las creencias populares en torno al poderoso influjo de la luna llena, Daquin asegura que «los primeros cuartos y los plenilunios son los puntos que ejercen menos influencia en la reiteración de los arrebatos de locura», ya que sus pacientes, "en esas etapas, estaban menos locos, más tranquilos y razonaban como si no hubieran tenido la mente alienada» (Daquin, 1791, p. 85-86). Excepción importante a esta regla general es la que representa la epilepsia, que sí recibía el influjo más directo de la luna llena. Merece la pena señalar la observación descrita por Daquin de un paciente melancólico y epiléptico:

En el hospital de locos a mi cargo pude observar a un loco que también era epiléptico y sobre quien el influjo de la luna afectaba a sus ataques epilépticos. Su locura triste, sombría y melancólica, era una simple alienación mental; pero la mayor desgracia de este individuo, digno de la mayor compasión; era que padecía todo a la vez, influyendo los puntos lunares afirmativos en la locura y los puntos lunares negativos en sus ataques epilépticos; quiero decir que, evidentemente, sufría también la influencia de las fases lunares más neutras (Daquin, 1791, p. 88).

Finalmente, no todos los tipos de locura estarían sujetos al dominio lunar de la misma manera. Las locuras curables o susceptibles de curación dependerían de la Luna mucho más que las incurables.

Estas ideas recuerdan, en cierto modo, la teoría hipocrática - retomada por Galeno y el galenismo medieval (Cooper, 2013) - de los días críticos, según la cual las crisis y las recaídas de determinadas enfermedades tenían lugar transcurridos periodos temporales fijos. Asimismo, esta concepción "meteorológica» y, por extensión, «ambientalista» de la salud y de la enfermedad se relaciona directamente con la larga tradición hipocrática inaugurada por Sobre Aires, Aguas y Lugares y que explica el marco doctrinal de otras obras de Daquin, como su importante topografía médica de la región de Chambéry (Daquin, 1787), y otras obras sobre los efectos beneficiosos de las aguas termales (Daquin, 1772; 1777; 1808), objeto de reciente interés historiográfico (Carpanetto, 2008). 
En todo caso, lo que más interesa señalar aquí es que, más allá de la exactitud o validez de tales observaciones empíricas, no cabe duda que los argumentos de Daquin están muy lejos de lecturas mágicas o esotéricas y mucho más cerca del talante científico de Newton $-y$ sus estudios sobre la influencia de la luna en la amplitud y regularidad de las mareas-, de Descartes $-y$ su calendario solar y lunar $-y$, naturalmente, del ya mencionado Toaldo, cuya influencia intelectual y científica es, como ya hemos visto, expresamente reconocida. Dicho de otro modo, Daquin no se está refiriendo a la causa última de la locura, sino a su periodicidad, insistiendo en que ésta se produciría por un efecto puramente físico, de atracción o presión sobre la «sustancia medular» del cerebro y no por una «fuerza oculta». En definitiva, no era el supuesto poder cósmico de la Luna la que influía en la locura, sino el efecto del ambiente, de la naturaleza, sobre la sensibilidad del cerebro (Stagnaro, 2013, p. XVIII).

El cerebro aparece, como es lógico, como el órgano de asiento de la locura, así lo expresa Daquin cuando asegura que «el cerebro y el origen de los nervios son los más comúnmente afectados en la locura» (Daquin, 1791, p. 16). Por eso insiste, cambiando de registro, en la utilidad de las autopsias y los estudios post mortem pues, si bien entiende que la disección de cadáveres aun no había proporcionado información suficiente sobre las causas de la locura, considera que la búsqueda de la lesión debe formar parte de las prioridades de los médicos interesados por la locura. Entre las causas físicas de la misma, le parece importante localizar e identificar las alteraciones orgánicas del cerebro, bien por inflamación de las fibras medulares o por cualquier tipo de compresión, bien porque son atacadas por la sequedad y la rigidez, bien por una excesiva debilidad y flaccidez (...), o incluso por la aparición de durezas en las membradas del cerebro» (Daquin, 1791, p. 16).

Llama la atención la importancia que Daquin otorga a las alteraciones vasculares del cerebro (con vasos sanguíneos unas veces endurecidos y estenosados; otras dilatados y varicosos), pero sobre todo a las alteraciones de los senos y los ventrículos -que pueden estar "llenos de sangre negruzca» u ocupados por hidátides- y de las membranas encefálicas: «la duramadre está tuberosa y putrefacta; la piamadre, callosa, con doble espesor o triple de lo que debe ser» (Daquin, 1791, p. 42). Esta alusión tan directa a las meninges parece preparar el terreno de lo que, pocas décadas después y de la mano de Antoine-Laurent-Jessé Bayle y sus estudios sobre la arac- noiditis crónica en pacientes con Parálisis General Progesiva (Bayle, 1822; 1826), supondría la irrupción definitiva de la lesión anatómica en la comprensión de la enfermedad mental (Brown, 1994; Huertas, 2005, p. 23 y ss). De igual modo, Daquin advierte de la relación entre la locura y determinadas anomalías del cráneo, como el excesivo grosor de los huesos, el grado de osificación, la conformación de las suturas o la mayor o menor curvatura de la calota (Daquin, 1791, p. 42). No cabe duda que, a este respecto, se sitúa en la misma línea de pensamiento que asumirá Pinel (1801, p. 106 y ss) en la Sección III de su Traité, dedicado a "Recherches anatomiques sur les vices de conformation du crâne des Aliénés».

En suma, junto a un cierto empirismo clínico, la búsqueda de la lesión en los enfermos mentales es una característica importante del proyecto científico de Daquin. Aun reconociendo que «a pesar de los progresos conseguidos [por la anatomía] en este siglo, no se ha ofrecido soluciones para las causas de la locura» (Daquin, 1791, p. 43), se muestra partidario de extender la práctica de la autopsia, insistiendo en las posibilidades que los hallazgos anatómicos podrían ofrecer para una mejor comprensión de la locura:

¡Cuán deseable sería que la anatomía pudiese descubrir en el cerebro las distintas lesiones que causan las diferentes clases de locura y cuáles son las partes alteradas tanto en el loco furioso como en el tranquilo, tanto en el extravagante como en el insensato, tanto en el imbécil como en el que simplemente está demente! (Daquin, 1791, p. 43).

Téngase en cuenta que la obra de Daquin es posterior a la de Morgagni, De sedibus et causis morborum per anatomen indagatis (1761), pero anterior a la Anatomie générale (1801) de Bichat. No es de extrañar, pues, que en La Philosophie de la folie, podamos identificar una voluntad de investigación anatomopatológica desde el convencimiento de que los hallazgos necrópticos debía ser útiles para entender los mecanismos fisiopatológicos y los síntomas clínicos en los enfermos vivos (Zampieri, Zanatta y Thiene, 2014), sin que aún haya cristalizado la estructura teórica que, a partir de los estudios de Bichat, Corvisat o Laënnec, dio lugar a la llamada mentalidad anatomoclínica (Laín, 1950). Parece evidente que el pensamiento de Daquin, a pesar de su enfoque "filosófico», tiene una fundamentación "médica» innegable e imprescindible, en un sentido muy similar al que años más tarde argumentarán los principales representantes del movimiento alienista (Peset, 1993; Huertas, 1996). 


\section{LOS PROLEGÓMENOS DEL TRATAMIENTO MORAL}

Desde el punto de vista del tratamiento, Daquin recomienda, aunque con mucha cautela, medios físicos tradicionales, así, considera que las sangría repetidas puedan agravar el pronóstico de la locura, prefiere los purgantes a los eméticos, y opina que el opio, en forma de láudano, y el alcanfor pueden ser adecuados por sus efectos narcóticos, aunque advierte que su eficacia depende mucho de la respuesta individual, siendo eficaz en unos pacientes y no en otros. Al igual que Pinel, desconfía del eléboro, y recomienda los baños, fríos o calientes según los casos. Sin embargo, considera que estos auxilios resultan muy limitados e insuficientes si no se añaden otros como el ejercicio, la dieta, la higiene, etc., concluyendo en que la higiene del alma es «la única que debe influir sobre la mente de estos enfermos, más que todos los agentes físicos empleados hasta el momento» (Daquin, 1791, p. 69).

El régimen de vida se convierte en el gran recurso terapéutico de Daquin. La recuperación de la tradición hipocrática y del poder sanador de la naturaleza - la vis medicatrix naturae o la natura morborum curatrix - es muy evidente en el pensamiento de Daquin, para el que el médico no ha de cumplir más que un papel de servidor o colaborador de dicha naturaleza -medicus autem naturae minister-. Termina desaconsejando las pócimas, fórmulas y demás remedios de la farmacopea para indicar al práctico que:

se dirija (al paciente mental) con un espíritu observador, pendiente del progreso de la naturaleza, a fin de favorecerla, de ayudarla en sus pasos, cuando se sitúa ya en el buen camino, y que retroceda cuando se extravía; quiero que el médico se acerque con parsimonia instruida y reflexionada, que no recete en la primera visita del enfermo medicamentos enérgicos y demoledores incluso antes de que la enfermedad se haya desarrollado y, sobre todo, que no ofrezca, con la cabeza baja, remedios nuevos, cuyo mérito consiste en anunciarse en los papeles públicos y cuya eficacia estriba en dar dinero a esos voceros y falsos que se llaman inventores (Daquin, 1791, p. 99).

Prudencia hipocrática e ilustrada, pocos medicamentos y mucha dieta, en el amplio sentido del concepto; esto es, como régimen de vida, como una higiene del alma en la que resultará imprescindible la dulzura en el trato. Su espíritu filantrópico le lleva a insistir en que es necesario observar y escuchar a los locos. Estamos aún lejos de una clínica de la escucha (Rigolí, 2001), ni siquiera del atisbo de una semiología de la subjetividad (Huertas, 2014), pero no cabe duda que, aunque su objetivo sea no tanto entenderles sino compadecerse de su infortunio, el contacto estrecho con los pacientes resultaría imprescindible para abordar el tratamiento de la locura.

Aun así, reconoce la necesidad de disciplina y advierte de la existencia de un punto conflictivo en la actitud a seguir ante los pacientes: «Para la curación de los locos debe existir (...) un término medio entre contrariar el objeto de su locura y lisonjearla» (Daquin, 1791, p. 51), que le aleja de Pinel para el que combatir la idea delirante es objeto prioritario del tratamiento.

Finalmente, en lo que se refiere al confinamiento de los locos, asegura taxativamente que «no se les debería encerrar», aseveración que se refiere exclusivamente al aislamiento celular ya que aboga por amplios espacios bordeados de muros, en los que personas cariñosas - «aunque fuerte y robustas, a fin de protegerles de su malicia, de sus caprichos y de sus violencias» (Daquin, 1791, p. 52-53) - estuviesen dispuestas a acompañarlos siempre y a vigilarlos constantemente. Recuérdese que, aunque procedente de una tradición asistencial diferente, el papel atribuido al personal auxiliar para que acompañe, persuada y controle sin ejercer coerción explícita sobre los pacientes, fue fundamental en la formulación del nonrestraint por parte de los británicos Robert Gardiner Hill (1839) y John Conolly (1856).

En la obra de Daquin se pueden identificar, pues, de manera sintética muchos de los principios de lo que muy poco tiempo se denominaría «tratamiento moral». El aislamiento, pero también, la persuasión por el razonamiento, la dulzura en el trato, el régimen de vida, etc., permitirían manejar las pasiones. Si la locura tenía una causalidad moral, el tratamiento debía perseguir el alivio moral de los pacientes. Resulta interesante constatar que de las tres ediciones existentes de La Philosophie de la folie, la primera (1971) no tuvo subtítulo alguno; la segunda (1972) se tituló La Philosophie de la Folie ou Essai Philosophique sur le traitement des personnes attaquées de Folie, lo que indica la importancia que el autor otorgaba a los aspectos terapéuticos, pero especialmente significativo es el título secundario de la tercera edición (1804): Où l'on prouve que cette maladie doit plutôt être traitée par les secours moraux que par les secours physiques. No olvidemos que esta última impresión es posterior al Traité de Pinel, no siendo descabellado pensar que la alusión directa -y programática- al tratamiento moral, ya desde la propia cabecera de la obra, tuviera una voluntad de confluir 
y asimilarse a la obra del parisino. Como ya se ha indicado, es precisamente esta edición la que Daquin dedicó a Pinel, a la vez que le reprochaba su silencio.

El tratamieto moral tuvo, como se sabe, unos desarrollos diversos que, en Francia, alcanzaron probablemente su máxima expresión con la obra de François Leuret (1840) y los debates que esta suscitó (Huertas, 2001), pero lo que más interesa aquí es que el proyecto terapéutico de Daquin es muy similar, aunque con ciertas diferencias y matices, con el que nos encontramos en la obra de Ph. Pinel. Para Pinel, hijo de la Revolución, su «tratamiento moral» tiene mucho que ver con la «incorruptibilidad moral» de Robespierre, siendo su objetivo fundamental devolver al paciente su «responsabilidad moral», convencerle de su error y propiciar el retorno a su anterior identidad individual y social (Huertas, 1990, p.399)

Pinel, al igual que Daquin, no es partidario de la utilización profusa e indiscriminada de fármacos (purgantes, eméticos, etc.) que constituían una farmacia gótica poco efectiva e, incluso, perjudicial. Advierte, por ejemplo, que el eléboro «puede producir a veces superpurgaciones violentas, vómitos pertinaces, convulsiones, inflamaciones de los intestinos, y aún la misma muerte (...) su administración se reducía a un ciego empirismo, ya que no estribaba en ningún fundamento sólido" (Pinel, 1801, p. 265). Y, al igual que Daquin, su hipocratismo le lleva a subrayar el carácter expectante que debe tener el acto médico, su potencial como ciencia de la observación y su confianza en el poder de la naturaleza.

Pero si en los primeros alienistas, y en general en la medicina ilustrada (Williams, 2002), puede identificarse un neohipocratismo muy evidente, hasta el punto de llegar a hablarse de "La revolución hipocrática de Philippe Pinel» (Peset, 2003), otras facetas del pensamiento clásico son recuperadas y reinterpretadas en esta época. La Ilustración resucita el estoicismo para encontrar una ética laica que sustituyese a la cristiana. En vez de leyes independientes, el philosophe busca guía y sentido en sí mismo, siendo un ser afectivo y racional, ya no un anima con solo elección racional. La ley natural ya no puede tan solo basarse en preceptos, en principios racionales, sino también en sensaciones, sentimientos y pasiones. Algunas ideas estoicas son fundamentales en la llustración, así el miedo a las pasiones, que deben ser dominadas. Se debe vivir de acuerdo con uno mismo, de acuerdo con la naturaleza. El estoico es el modelo del philosophe francés, pues cree en la sabiduría y en la salvación de los pueblos. Esta concepción estoica del siglo XVIII tiene dos rasgos distintivos: el primero, es la insistencia en el autodominio; el segundo, la consideración del ser humano como parte integral de la naturaleza, desde el ámbito familiar y el compañerismo hasta la especie humana. Se pretende justicia para toda la humanidad, así como una visión integradora de cuerpo y alma. Virtud, sabiduría y naturaleza quedan igualadas. La salud es acuerdo y obediencia a la naturaleza y, por tanto, con la sabiduría y con dios (Peset, 2003, p. 264).

En suma, el «hipocratismo ilustrado» de Joseph Daquin, compartido por otros autores $-y$ por Pinel de manera sobresaliente-, aparece como un elemento imprescindible, yo diría que inherente, en el nacimiento del alienismo que viene a completar, en buena medida, las visiones que en los últimos tiempos se han ofrecido de este proceso.

\section{DAQUIN VERSUS PINEL}

Con todo, merece la pena señalar cómo, en los años centrales del siglo XIX, el debate sobre quien fue el «creador de la ciencia de las enfermedades mentales» ocupó un cierto espacio en la literatura psiquiátrica con motivo de la publicación de una Notice biographique sur le médecin Daquin. Su autor, Louis Guilland (1852) reclama la prioridad de las ideas de Daquin sobre las de Pinel con diversos argumentos; por un lado, hace notar el reconocimiento internacional de la obra del médico saboyano, recogiendo una frase del italiano Antonio Galloni, primer director del manicomio de San Lazzaro en Reggio Emilia (Mazza, 1975), en la que considera al «ilustre Daquin, predecesor de Pinel» (Guilland, 1852: 22). Pero, por otro lado, arremete contra Pinel lamentado su ya comentado silencio con respecto a la obra de Daquin y, sobre todo, dejando en el aire la duda sobre la originalidad de su obra. Sin llegar a acusarle de plagio, se pregunta «¿Por qué Pinel, que en el curso de su obra atribuye el nombre de manie a una especie particular de alienación, titula incorrectamente su Traité médico-philosophique sur la manie? ¿No tendría la idea del título más natural de Daquin Philosophie de la Folie, pero desistió ante una coincidencia comprometedora?» (Guilland, 1852: 22).

Como no podía ser de otro modo, la Notice de GuiIland no tardó en ser contestada desde el seno del alienismo francés. Brierre de Boismont (1797-1881), en una conferencia pronunciada en la Société médicale d'émulation y publicada en los Annales médicopsychologiques, analiza la cuestión de la prioridad del «descubrimiento» del tratamiento moral. Lo primero que argumenta es que, en lugar de disputar sobre dicha prioridad, lo primero que habría que hacer, pres- 
cindiendo de intereses nacionalistas, es estudiar los textos y compararlos (Brierre de Boismont, 1854, p. 2 ). De su lectura detenida de las dos obras, Brierre concluye en que, aun aceptando que Daquin precedió a Pinel en la puesta en marcha del llamado tratamiento moral y los mismos o muy parecidos proyectos de reforma asistencial, «el espíritu filosófico, la altura de miras, la firmeza en la ejecución, otorgan al Tratado del médico francés un valor que no tiene La Philosophie de la Folie» (Brierre de Boismont, 1854, p. 10).

Aun así, Brierre no deja de lamentar el olvido al que había sido sometido la obra de Daquin:

\footnotetext{
se experimenta un doloroso asombro al ver la primera edición de Daquin, impresa en lengua francesa y a las puertas de Francia, ignorada por Pinel que cita a otros autores antiguos y contemporáneos. Esta sorpresa aumenta cuando se constata el mismo silencio en la edición de 1809, en la Clinique de la Salpêtrière de 1807, y en las seis ediciones de la Nosographie, la última de 1818, máxime cuando Daquin había dedicado a Pinel la segunda edición de 1804, cinco años antes de la segunda edición del Traité médico-philosophique sur l'aliénation (Brierre de Boismont, 1854, p. 12)
}

El profundo conocimiento que Brierre de Boismont demuestra tener de la obra de Pinel, a la que considera de mayor calidad y trascendencia que la de Daquin, no le impiden dedicar una agria crítica al "padre» del alienismo francés: «¿será verdad que en el corazón de los hombres más ilustres hay lugares secretos en los que se ocultan las debilidades de nuestra naturaleza (...) aquellas debilidades que imposibilitan pronunciar o escribir el nombre el rival, aquella que un autor moderno ha llamado la conspiración del silencio?» (Brierre de Boismont, 1854, p. 13). Duras palabras, sin duda, y especialmente significativas si tenemos en cuenta que quien las estaba formulando era ya, en ese momento, una de las figuras más representativas del alienismo francés, llegando a ser co-editor de los Annales médico-psychologiques y presidente de la Société Médico-Psychologique, además de autor de obras relevantes, que le permitieron adquirir un gran prestigio internacional ${ }^{4}$.

En contraste, no podemos dejar de mencionar aquí la actitud opuesta que Brierre de Boismont mantuvo años más tarde, en 1864, con el psiquiatra italiano Carlo Livi (1823-1876), facultativo del manicomio de Siena y profesor de Medicina Legal e Higiene en su universidad. Livi había publicado Frenologia forense. Delle frenopatie considerate relativamente alla medi- cina legale (1863), que fue objeto de la crítica de Brierre de Boismont en la sesión del 29 de junio de 1863 de la Société médico-psychologique, en los siguientes términos:

Nos desagrada encontrar en este trabajo... una frase que podría ser ofensiva para Francia, si no la achacáramos a la efervescencia del amor patrio. El señor Livi hablando de la reforma de la asistencia a los locos, reclama en términos bastante vivos este honor para Vincenzo Chiarugi, quien la habría anunciado ocho años antes que Pinel en su Trattato della Pazzia publicado en Florencia en 1793. Él cita, además, a otro médico italiano, Daquin, quien escribió la Philosophie de la folie en 1791. El señor Livi habría podido añadir también el nombre de Tuke, quien en 1792 hacía público su primer programa sobre el asilo de York, pero ¿̇en qué disminuye, como siempre diremos, la gloria de Pinel, si algún hombre de bien tuvo, contemporáneamente a él o algo antes, el pensamiento de mejorar la suerte de los locos y lo llevó a cabo? Mencionar sus nombres es un acto de justicia: pero se debe reconocer que el genio de Pinel ha fecundado la idea y la ha introducido (aquí está lo importante) en la práctica (Brierre de Boismont, 1863, p. 452).

Las aguas vuelven a su cauce, si es que alguna vez se salieron. El gran Pinel y su obra aparecen, como no podía ser de otro modo, en la cúspide del alienismo y el desagravio a Daquin queda ahora suficientemente matizado. Sin embargo, la réplica de Livi no se hizo esperar y en una "Carta abierta a Brierre de Boismont», publicada en La Nazione, reivindica a Chiarugi e insiste en que la acción filantrópica y liberadora de los locos en San Bonifacio de Florencia había sido previa a la de Bicêtre, y en que las ideas vertidas en Della pazzia resultaban, asimismo, pioneras en relación a las aportaciones posteriores del francés ${ }^{5}$. No es este el momento de analizar las aportaciones de Chiarugi, pues en este mismo dossier hay otro artículo dedicado a este importante autor, pero sí merece la pena consignar la diferente consideración que para el alienismo francés tuvieron Daquin y Chiarugi pues, aunque los argumentos se repiten: «Pinel toma mucho de la obra de Chiarugi sin dignarse nunca a citar ni siquiera su nombre» (Livi, [1864] 2004, p. 119), el agravio comparativo entre uno y otro resulta evidente para Livi, cuando interpela a Brierre diciéndole que: «la justicia que Vos no negasteis hace diez años al súbdito italiano de Chambèry, ¿ por qué negársela hoy al ciudadano italiano de Florencia? ¿Quizás presentíais que Saboya un día ya no sería nuestra? Yo nunca cometería el error de creeros profeta» (Livi, [1864] 2004, p. 121). 
En todo caso, y a pesar de polémicas y desagravios, lo cierto es que la obra de Daquin fue totalmente ignorada por los alienistas franceses. Ni Pinel, como hemos visto, ni Esquirol le citaron una sola vez e, incluso, Casimir Pinel, según nos cuenta Claude Quétel, llegó a afirmar que "La Philosophie de la Folie es una obra que nueve de cada diez alienistas no han leído, y que no se encuentra ni en las librerías, ni en la biblioteca de la Facultad», lo que no deja de resultar, según el propio Quétel, al menos curioso para una obra que había conocido tres ediciones, todas en francés, una de ellas publicada en París (Quétel, 1987: 27).

Al margen de disputas con mayor o menor contenido nacionalista, no cabe duda que la figura de Pinel se ha mantenido como referente absoluto del nacimiento del alienismo. Un tópico historiográfico al que no ha sido ajeno todo un movimiento de "devoción extrema» - de bondieuserie laica- hacia Philippe Pinel que, orquestado y mantenido en el tiempo por autores como Casimir Pinel (1859), editor de la correspondencia de su tio Philippe Pinel (Peset, 1991), o como René Semeaigne (1888; 1930), ha construido una hagiografía que, rozando el mito, tendió a silenciar e invalidar los logros de sus contemporáneos. Una historiografía que ha alimentado durante mucho tiempo el «mito fundacional» del alienismo ligado al conocido «gesto» liberador de Pinel (Gourevitch, 1991; Weiner, 1994a). Sin embargo, como es bien conocido, diversos autores han contribuido, en las últimas décadas, a reinterpretar la obra pineliana y su influencia. No pocos trabajos, como los de Michel Foucault (1961), Gladys Swain (1977), Jan Goldstein (1987), o Dora Weiner (1999), entre otros, han ofrecido una visión diferente de ese momento crucial de la historia de la psiquiatría. Hoy sabemos que Joseph Daquin en Chambèry, William Battie en Londres, Vincenzo Chiarugi en Florencia, William Tuke en York, Pinel en Paris, etc., desarrollaron en sus respectivas instituciones y establecimientos una labor médica y humanitaria que, desde luego, no puede atribuirse a nadie en exclusiva, sino a todo un proceso que se imbrica directamente con la revolución liberal en sus diversas versiones nacionales, resultando de gran interés analizar sus características propias y sus rasgos diferenciales.

Sin embargo, tampoco debe olvidarse que si las reformas de Pinel fueron similares y comparables con las de Daquin y otros alienistas contemporáneos unas reformas para las que el parisino contó con la inestimable colaboración e, incluso, con el magisterio del celador Jean-Baptiste Pussin (Weiner, 1994b)-, su aportación específica a la clínica es muy superior a la del médico saboyano. La clínica como "camino consciente y sistemático» (Bercherie, 1980, p. 15), que requería del método analítico de Condillac, de la historia natural como modelo de investigación y de la observación hipocrática para la descripción y clasificación de las enfermedades.

De lo que no cabe duda es que el fin de la luces crea un caldo de cultivo intelectual (ilustrado y filantrópico) que marca una nueva forma de entender la locura y de actuar sobre el loco. La llustración y el posterior Romanticismo, propiciaron una nueva percepción del individuo que condujo, a su vez, a una nueva visión de la locura, decisiva en la creación de nuevas instituciones para locos y en la consolidación de un discurso científico-médico en torno a las enfermedades mentales (Kaufmann, 1995; Novella, 2013). Este interés por el estudio del hombre (del ser humano) y, en particular, del hombre "alterado» dio lugar primero a las teorías sobre la locura, después a la preocupación por la asistencia al loco y, más tarde, a la teoría psiquiátrica propiamente dicha. La decadencia de la vieja monarquía, la joven revolución y el brillante Imperio son etapas paralelas a estas novedades. El ciudadano Daquin y el ciudadano Pinel coincidieron con mayor o menor fortuna en aquella etapa revolucionaria y fundacional de la psiquiatría.

\section{NOTAS}

1 Daquin, Joseph (1791). La Philosophie de la folie, Chambéry, Gorrin. El mismo texto fue publicado un año más tarde, en 1792, con el título La Philosophie de la folie, ou Essai Philosophique sur le traitement des personnes attaquées de Folie, París, Chez Nee de la Rochelle. Finalmente, en 1804 apareció una segunda edición, algo más ampliada y matizada, titulada La Philosophie de la folie, Où l'on prouve que cette maladie doit plutôt être traitée par les secours moraux que par les secours physiques, Chambéry, De l'imprimerie Cléaz. Posteriormente, la obra se ha vuelto a editar en francés, publicada por Frénésie

Éditions en 1987, con una Presentación de Claude Quétel y en castellano con dos ediciones, una publicada por la Asociación Española de Neuropsiquiatría (AEN) en 2000, que agrupa el texto de Esquirol, Sobre las pasiones, y el de Daquin, y cuenta con una Introducción de José Luis Peset; la otra, más reciente, ha sido editada en 2013 por Polemos (Buenos Aires), en un volumen que, con el título genérico de Los prolegómenos del tratamiento moral, agrupa obras de William Battie, Hohn Monro, Jean Colombier y François Doublet, Joseph Daquin y Alexander Crichton. Cuenta con una introducción de Juan Carlos Stagnaro. 
2 La vinculación de la Baja Saboya a Francia y a Italia, según la época que se considere, ha dado lugar a ciertas reivindicaciones de nacionalismo científico en torno a la figura de Daquin. Sin embargo, Daquin siempre firmó sus obras como Joseph, y no como Giuseppe, y las publicó en francés.

3 Estas tres facultades del alma, definidas por los antiguos, fueron recogidas y modernizadas por Juan Huarte de San Juan; sobre el particular, véase Arquiola (1984) o Garrabé (2003).

\section{BIBLIOGRAFÍA}

Arquiola, Elvira (1984), “Biología y política en el Examen de Ingenios de Huarte de San Juan”, Asclepio, 36, pp. 85-121.

Bayle Antoine-Laurent-Jessé (1822), Recherches sur les maladies mentales. Paris, Didot.

Bayle, Antoine-Laurent-Jessé (1826), Traité des maladies du cerveau et de ses membranes. Paris, Chez Gabon.

Bercherie, Paul (1980), Les Fondements de la Clinique. Histoire et structure du savoir psychiatrique. Paris, Navarin.

Berrios, German y Dominique Beer (1994), "The notion of unitary psychosis: a conceptual history", History of Psychiatry, 5, pp. 13-26.

Berrios, German y M. Mohanna (1990), “Durkheim and French Psychiatric View on Suicide during the 19th Century. A conceptual history", British Journal of Psychiatry, 156, pp. 1-9.

Berrios, German y M. Mohanna (1995), "Suicidal Behaviour. Clinical Section." En: Berrios, German; Porter, Roy (ed.), A History of Clinical Psichiatry. The Origin and History of Psychiatric Disorders, Londres, Atholone, pp. 612-629.

Bichat, Xavier (An X - 1801), Anatomie générale appliquée à la physiologie et à la medicine. Paris, Brosson, Gabon et Cie.

Brierre de Boismont, Alexandre Jacques François (1854), “De la réforme du traitement des aliénés a l'occasion de la Notice biographique sur le médecin Daquin par M. le Dr. Guilland fils", Annales médico-psychologiques (2a serie), 6, pp. 2-13.

Bierre de Boismont, Alexandre Jacques François (1863), "Brierre de Boismont donne lectura d'un rapport sur la candidatura de M. Livi, au titre de membre asocié étranger", Annales médico-psychologiques, 2, pp. 541-459.

Brown, Edward (1994), "French Psychiatry's Initial Reception of Bayle's Discovery of General Paresis of the Insane", Bulletin of the History of Medicine, 68, pp. 235-253.

Caron, C. (1964), Joseph Daquin et les maladies mentaux en Savoie a la fin du 18eme siècle. Thèse médecine, Lyon.
4 Para España, puede verse Huertas y Novella (2011). También Novella y Huertas (2011)

5 Livi, Carlo (1864), Lettere al célèbre Dott. Al Brierre de Boismont, cavalieri della legione d'onore», La Nazione, sept. 1864. Reimpresa en 1927 en Rassegna di Studi Psichiatrici, 16: 214-233. Existe una traducción al castellano en Huertas y del Cura (2004). Se cita por esta última.

Carpanetto, Dino (2008), "Scienziate confini culturali: termalimoi Savoia nell'opera di Joseph Daquin (1732-1825)", Gesnerus, 65, pp. 157-175.

Charland, Louis (2008), "A moral line in the sand: Alexander Crichton and Philippe Pinel on the psycotathology of the passions". En: Charland, Louis y Zachar, Peter (eds.), Fact and Value in emotion. Amsterdan/Philadelphia, John Benjamin, pp. 15-33.

Chevrey, J. (1816), Essai médical sur le suicide: considéré, dans tous les cas, comme le résultat d'une aliénation mentale. Paris, Imp. Didot Jeune.

Conolly, John (1856), The treatment of the insane without mechanical restraints. London, Smith, Elder and Co.

Cooper, Glen M. (2013), "Approaches to the Critical Days in Late Medieval and Renaissance Thinkers", Early Science and Medicine, 18 (6), pp. 536-565.

Cuevas, Francisco (2006), "Una revisión de las ideas en torno al suicidio en el tránsito de la llustración al Romanticismo", Cuadernos de Ilustración y Romanticismo, 14, pp. 11-41.

Daquin, Joseph (1772), Analyse des eaux thermales d'Aix en Savoye. Chambéry, Gorrin.

Daquin, Joseph (1777), Analyse des prétendues eaux ferrugineuses de la Boisse, situées près de Chambéry. Chambéry, Lullin.

Daquin, Joseph (1787), Topographie médicale de la ville de Chambéry et de ses environs. Chambéry, Gorrin.

Daquin, Joseph (1791), La Philosophie de la folie. Chambéry, Gorrin.

Daquin, Joseph (1792), La Philosophie de la folie, ou Essai Philosophique sur le traitement des personnes attaquées de Folie. Paris, Chez Nee de la Rochelle.

Daquin, J. (An XII - 1804), La Philosophie de la folie, Où I'on prouve que cette maladie doit plutôt être traitée par les 
secours moraux que par les secours physiques. Chambéry, De l’imprimerie Cléaz.

Daquin, Joseph (1808), Des eaux thermales d'Aix dans le département du Mont-Blanc: de leurs vertus médicales. Chambéry, Cléaz.

Esquirol, Jean-Étienne-Dominique (1805), Des passions considérées comme causes, symptômes et moyens curatifs de l'aliénation mentale. Thèse de médecine, Paris.

Ferrio, L. (1954), “Un pioniere dell'assistenza psichiatrica: Giuseppe Daquin", Rivista di storia delle scienze mediche e naturali, 45, pp. 156-171.

Foucault, Michel (1961), Folie et déraison. Histoire de la folie à l'âge clasique. Paris, Plon.

Garrabé, Jean (2003), “Juan Huarte y el pensamiento humanista en Europa". En: Fuentenebro, F.; R. Huertas y C. Valiente (eds.), Historia de la psiquiatría en Europa. Temas y tendencias, Madrid, Frenia, pp. 51-63.

Gauchet, Marcel y Gladys Swain (1980), La pratique de l'esprit humaine: l'institution asilaire et la révolution démocratique. Paris, Gallimard, pp. 413-422.

Goldstein, Jan (1987), Console and classify. The French Psychiatric Profession in the Ninneteenth Century. Cambridge, Cambridge University Press.

Gourevitch, Michel (1991), "Pinel pere fondateur, mythes et réalites", L 'Evolution psychiatrique, 56, pp. 595-602.

Guilland, Louis (1852), Notice biographique sur le médecin Daquin. Chambéry, Chez Puthod.

Hill, Robert Gardiner (1839), Total Abolition of Personal Restraint in the Treatment of the Insane: A Lecture on the Management of Lunatic Asylums, and the Treatment of the Insane. Londres, Simpkin, Marshall, and S. Highley.

Huertas, Rafael (1990), "Medicina mental y revolución busquesa: sobre los orígenes de la asistencia psiquiátrica", Revista de la Asociación Española de Psiquiatría, 10 (43), pp. 389399.

Huertas, Rafael (1996), "L'alienismo e la mentalità anatomoclinica: L'opera di J.E.D. Esquirol”, Medicina nei secoli, 8, pp. 367-380

Huertas, Rafael (1999), “Nosografía y antinosografía en la psiquiatría del siglo XIX: en torno a la psicosis única", Revista de la Asociación Española de Neuropsiquiatría, 19 (69), pp. 63-76.

Huertas, Rafael (2001), "François Leuret: Terapia e intimidación moral”. En: Leuret, François. El tratamiento moral de la locura, Madrid, Asociación Española de Neuropsiquiatría, pp. 7-13.
Huertas, Rafael (2005), El siglo de la clínica. Para una teoría de la práctica psiquiátrica. Madrid, Frenia.

Huertas, Rafael (2008), "Between doctrine and clinical practice: nosography and semiology in the work of Jean-EtienneDominique Esquirol (1772-1840)", History of Psychiatry, 19 (2), pp. 123-140.

Huertas, Rafael (2014), "Subjectivity in clinical practice: on the origins of psychiatric semiology in early Franch alienism", History of Psychiatry, 25, pp. 454-467.

Huertas, Rafael y Mercedes del Cura (2004), "Chiarugi versus Pinel. Carta abierta de Carlo Livi a Brierre de Boismont", Frenia, 4 (2), pp. 109-133.

Huertas, Rafael y Enric Novella (2011), “L’aliénisme français et I'institutionnalisation du savoir psychiatrique en Espagne: I'affaire Sagrera (1863-1864)", L'Evolution Psychiatrique, 76 (3), pp. 537-547.

Kaufmann, Dora (1995), Aufklärung, Selbsterfahrung und die "Erfindung" der Psychiatrie in Deutschland, 1770-1850. Göttingen, Vandenhoeck \& Ruprecht.

Laín Entralgo, Pedro (1950), La historia clínica. Historia y teoría del relato patobiográfico. Madrid, CSIC.

Livi, Carlo (1863-1868), Frenologia forense. Delle frenopatie considerate relativamente alla medicina legale. Milano, Chiusi.

Leuret, François (1840), Du traitement moral de la folie. Paris, Ballière.

Livi, Carlo (1927), "Lettere al célèbre Dott. Al Brierre de Boismont, cavalieri della legione d'onore", Rassegna di Studi Psichiatrici, 16, pp. 214-233.

Martínez-Pérez, José (2001), "Suicidio, crisis política y medicina mental en la Francia del siglo XIX (1801-1885)", Frenia, 1 (2), pp. 39-65.

Mazza, A. (1975), "Antonio Galloni, 1o direttore del San Lazzaro", Rivista Sperimentale di freniatria e di medicina legale, 99, pp. 56-69.

Morgagni, Giovanni Battista (1761), De sedibus et causis morborum per anatomen indagatis. Venecia, ex Thypographia Remondiniana.

Novella, Enric (2013), La ciencia del alma. Locura y modernidad en la cultura española del siglo XIX. Madrid, Iberamericana/ Vervuert.

Novella, Enric y Rafael Huertas (2011), “Alexandre Brierre de Boismont and the origins of the Spanish psychiatric profession", History of Psychiatry, 22 (4), pp. 387-402.

Nyffeler, Johann R. (1961), Joseph Daquin und seine Philosophie de la folie. Zurich, Juris. 
Padovani, E. (1927), "Pinel e il rinnovamento dell'assistenza degli alienati: I suoi percusori: I predecessori italiani: Giuseppe Daquin e Vincenzo Chiarugi", Giornale di psichiatria e di neuropatología, 55, pp. 69-124.

Peset, José Luis (1991), "Política y educación en la correspondencia familiar de Philippe Pinel", Llull, 14, pp. 589-602.

Peset, José Luis (1993), Las heridas de la ciencia. Salamanca, Junta de Castilla y León.

Peset, José Luis (2000), “Daquin y Esquirol: el miedo a las pasiones". En: Esquirol, J.-E.-D. Sobre las pasiones \& Daquin, J. La Filosofía de las pasiones, Madrid, Asociación Española de Neuropsiquiatría, pp. 7-19.

Peset, José Luis (2003), “La revolución hipocrática de Philippe Pinel”, Asclepio, 55 (1), pp. 263-279.

Pinel, Casimir (1859), Lettres de Pinel, précédées d'une notice plus étendue sur sa vie par son neveu le Dr. Casimir Pinel. Paris, Victor Masson.

Pinel, Philippe (1801-An IX), Traité médico-philosophique sur l'aliénation mentale ou la manie. Paris, Richard, Caille et Ranvier.

Rigolí, Juan (2001), Lire le délire. Aliénisme, rhétorique et litterature en France au XIXe siécle. Paris, Fayard.

Semelaigne, René (1888), Philippe Pinel et son oeuvre. Au point de vue de la médecine mentale. Paris, Imprimerie Réunies.

Semelaigne, René (1930-32), Les pionniers de la psychiatrie française avant et après Pinel. Paris, Baillière.

Shorter, Edward (1997), A History of Psychiatry. New York, J. Wiley and Sons.

Stagnaro, Juan Carlos (2013), "Apuntes biográficos”. En: Los prolegómenos del tratamiento moral, Buenos Aires, Polemos, pp. IX-XXIII.

Swain, Gladys (1977), Le sujet de la folie. Naissance de la psychiatrie. Toulouse, Privat.
Toaldo, Giuseppe (1770), Della vera influenza degli astri, delle stagioni, e mutazioni di tempo, saggio meteorologico fondato sopra lunghe osservazioni, ed applicato agli usi dell'agricultura, medicina, nautica. Padova, Apresso Gio. Manfrè.

Toaldo, Giuseppe (1784), Essai météorologique sur la véritable influence des astres, des saisons et changemens de tems. Chambery, Gorrin.

Vanni, D; Salomone, D.; Pomini, D.; Vanni, P. and Ottaviani, R. (1999), “Joseph Daquin, Piedmontese Savoyard physician: a 'not well-known Chiarugi'”, Vesalius, 5 (1), pp. 30-40.

Weiner, Dora B. (1990), "Mind and body in the clinic: Philippe Pinel, Alexander Crichton, Dominique Esquirol, and the birth of psychiatry". En: Rousseau, G.S. (ed.), The languages of psyche: Mind and body in enlightenment though, Berkeley, University of California Press, pp. 331-402.

Weiner, Dora (1994a), "Le geste de Pinel: The History of a Psychiatric Myth". En: Micale, Mark, S.; Porter, Roy (eds.), Discovering the History of Psychiatry, New York-Oxford, Oxford University Press, pp. 232-247.

Weiner, Dora (1994b), "Pinel et Pussin à Bicêtre: causes et conséquences méthodologiques d'un rencontré". En: Garrabé, Jean (ed.), Philippe Pinel, Paris, Les êmpecheurs de penser en rond, pp. 95-116.

Weiner, D. B. (1999), Comprendre et soigner. Philippe Pinel (1745-1826), la médecine de l'esprit. Paris, Fayard.

Williams, E.A. (2002), "Hippocrates and the Montpellier Vitalist in the French Medical Enlightement". En: Cantor, D. (ed.), Reinventing Hippocrates, Aldershot, Asghate, pp. 157-177.

Zampieri, Fabio; Alberto Zanatta y Gaetano Thiene (2014), “An etymological 'autopsy of Morgagni's title: De sedibus et causis morborum per anatomen indagatis (1761)", Human Pathology, 45 (1), pp. 12-16. 